Main topic

\title{
Pediatric trauma
}

\author{
Arnold G. Coran \\ Section of Pediatric Surgery University of Michigan Medical School, C. S. Mott Children's Hospital, Ann Arber, MI 48109, USA
}

The main topic in this issue of Pediatric Surgery International is devoted to pediatric trauma. Trauma is now the leading case of death in all children between the ages of 1 and 14 years in the United States and a major cause of mortality throughout the rest of the world. This has resulted in a major resurgence of interest in this area among pediatric surgeons. This recent interest is evidenced by the establishment of the National Conference on Pediatric Trauma, which held its first two meetings in Boston, Massachusetts, in 1985 and 1987. The Third National Conference on Pediatric Trauma was recently held in Ann Arbor, Michigan, on 21-23 September 1989. Several original papers were presented during that $21 / 2$-day conference. The three articles published in this issue were presented at this last conference and represent significant new information in the field of pediatric trauma, which I feel should be disseminated to pediatric surgeons throughout the world. The first article, "The hormonal and metabolic response to stress in the neonate" by Schmeling and Coran, is a comprehensive review of all the information available in the world literature on this important subject. Detaild knowledge of the metabolic response of the neonate to operative and nonoperative trauma is critical in fine-tuning the management of sick infants. This review points out how little is known in this area and how much further research needs to be done. The second article, "Extracorporeal life support (ECLS) for pediatric trauma: experience with five cases" by Anderson et al., describes a unique experience with a group of pediatric trauma patients requiring extracorporeal membrane oxygenation (ECMO) for management of their severe pulmonary insufficiency. This represents a new application of ECMO, which has been extremely successful in the management of neonatal respiratory distress. Finally, the third article, "Successful early management of adolescent inhalation injuries: a preliminary report" by Fallot and Longmire-Cooke, represents an unusual experience with the management of inhalation injuries in children and describes a new approach to this very serious problem. These three articles will hopefully provide new and significant information to pediatric surgeons involved in the care of pediatric trauma victims. 